\title{
Bilateral Bochdalek hernias associated with severe aortic tortuosity and aberrant right subclavian artery
}

\author{
Sinan Akay ${ }^{1}$, MD, Bilal Battal ${ }^{1}$, MD, Kemal Kara' ${ }^{1}$,MD, Ugur Bozlar ${ }^{1}$, MD
}

\begin{abstract}
Although Bochdalek hernia is the most common congenital diaphragmatic hernia in adults, bilateral occurrence is rare. To the best of our knowledge, only case reports on Bochdalek hernia and its associated pathologies have been previously published. Herein, we present the case of a 27-year-old man with bilateral Bochdalek hernias, which were detected incidentally. The bilateral Bochdalek hernias were found to be associated with severe aortic tortuosity and aberrant right subclavian artery.
\end{abstract}

Keywords: aorta, Bochdalek hernia, computed tomography angiography, loop, tortuosity

\section{INTRODUCTION}

The most common congenital diaphragmatic hernia $(\mathrm{CDH})$ observed in adults is Bochdalek hernia $(\mathrm{BH})$, with a prevalence of $0.17 \%-6 \% .^{(1,2)}$ Although it has been described to occur more commonly on the left side of the body, other series have found a higher incidence on the right. ${ }^{(1,3,4)}$ While there are many reports ${ }^{(2,3)}$ on the incidence of $\mathrm{BH}$, the side of the body in which $\mathrm{BH}$ occurs, the demographics of patients with $\mathrm{BH}$, and its associated pathologies are not well described. Also, bilaterality of $\mathrm{BH}$ has seldom been mentioned in the literature, ${ }^{(1,3,4)}$ although its incidence has been reported to be $3 \%-6 \%$ by Gale. ${ }^{(2)}$ In this report, we describe a case of bilateral $\mathrm{BHs}$, which were incidentally discovered and found to be associated with severe aortic elongation and an aberrant right subclavian artery. To the best of our knowledge, this is the first case report that suggests a link between vascular anomalies and $\mathrm{BH}$.

\section{CASE REPORT}

A 27-year-old man presented to the pulmonary medicine department of our hospital with complaints of shortness of breath following exercise and claimed that he had been suffering from this distressing reaction since childhood. Chest radiography showed an increased cardiothoracic ratio, an abnormally prominent aortic knuckle, decreased volume of the left lung, and a leftward deviation of the mediastinal structures and heart. In addition, lobulated diaphragmatic contours, an obliterated left costodiaphragmatic recess and a loop formation that was superimposed on the heart shadow at the distal segment of the descending thoracic aorta, were observed (Fig. 1). Computed tomography angiography (CTA) was performed to rule out aortic aneurysm; it revealed a severely elongated thoracic aorta, with two loop formations at the level of the proximal and distal segments

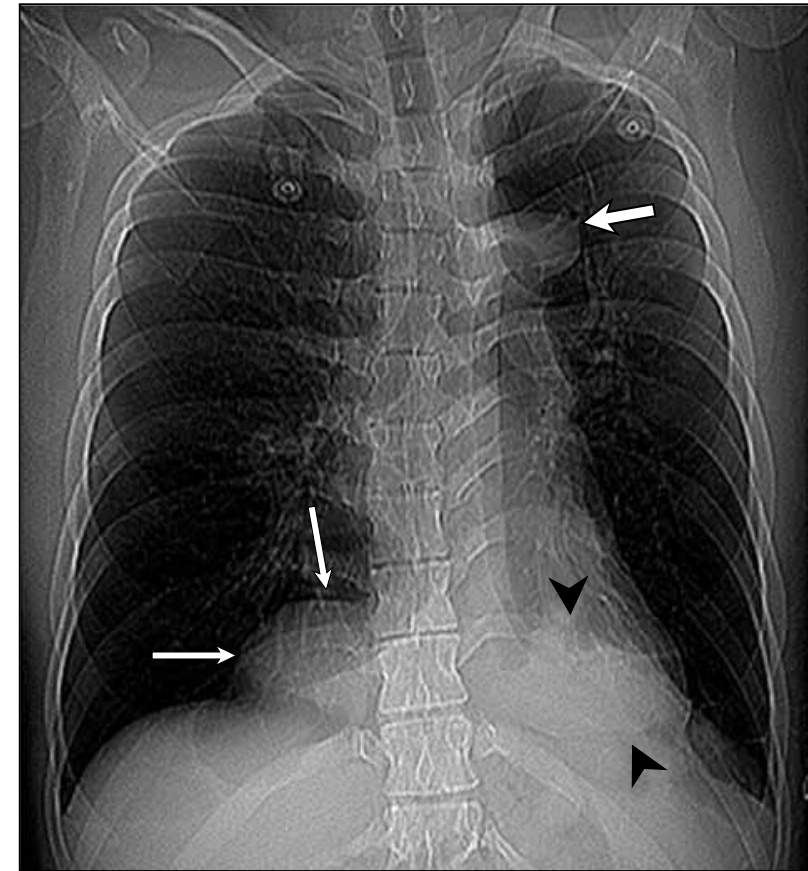

Fig. 1 CT angiography scanogram shows a prominent aortic knuckle (thick white arrow), an increased cardiothoracic ratio, decreased left lung volume, a leftward deviation of the mediastinal structures and heart, an obliterated left costodiaphragmatic recess, and lobulated diaphragmatic contours (thin white arrows). A loop formation that was superimposed on the heart shadow at the distal segment of the descending thoracic aorta is also seen (black arrowheads).

of the descending aorta, and an associated aberrant right subclavian artery. Bilateral BHs were also detected with CTA. The following were also observed: herniated left kidney; abdominal fat tissue that extends into the left hemithorax; and herniated segments 2 and $4 \mathrm{~A}$ of the liver that extends into the right hemithorax through the bilateral diaphragmatic defects (Fig. 2). The pulmonary venous system, inferior and superior vena cava, and other arterial structures were normal. 

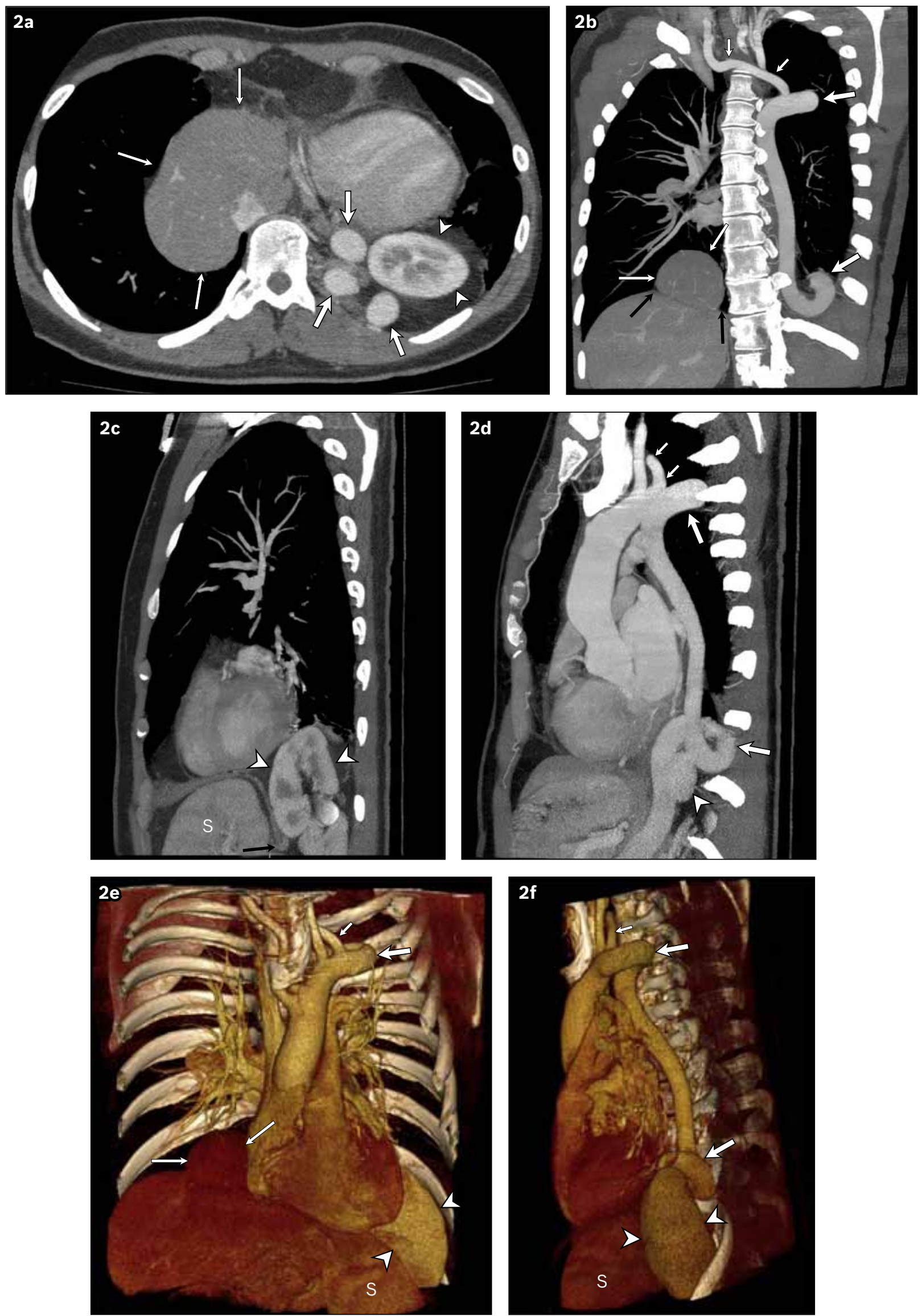

Fig. 2 (a) Axial, (b) coronal, (c) sagittal, (d) oblique-sagittal, and maximum-intensity-projection and volume-rendered (e) coronal and (f) oblique-sagittal computed tomography angiography images demonstrate the herniating part of segments 2 and $4 \mathrm{~A}$ of the liver (thin white arrows), herniating left kidney (white arrowheads), and a small portion of abdominal fat tissue from the bilateral diaphragmatic defects (black arrows). A severely elongated thoracic aorta, with two loop formations at the level of the proximal and distal segments of the descending aorta (thick white arrows), and an aberrant right subclavian artery (short white arrows) are also seen. S: spleen 


\section{DISCUSSION}

$\mathrm{BH}$ is the leading form of $\mathrm{CDH}$, with a prevalence in the adult population ranging from $0.17 \%$ to $6 \% .^{(1,2)} \mathrm{BH}$ is usually detected in newborns, where it presents as acute respiratory distress, or later in infancy, where it presents as respiratory insufficiency. ${ }^{(5)}$ The patient's prognosis is poor if the hernia is large, with mortality rates as high as $30 \%$ being the norm; death results from hypoplasia of the underlying lung and pulmonary arterial hypertension. ${ }^{(6)}$ However, as small hernias are usually asymptomatic, they can be missed and diagnosed only in adulthood. (7) Most $\mathrm{BH}$ cases in adults are discovered incidentally via the use of imaging modalities during investigations for other pathologies. Such cases of $\mathrm{BH}$ are likely to be asymptomatic. ${ }^{(8)}$ Although hernias in the posterior part of the diaphragm are thought to be congenital, such hernias may also be acquired or exacerbated through trauma or physical exercise. ${ }^{(9)}$

BHs can present on radiographic imaging as a focal bulge in the hemidiaphragm or a mass-like opacity adjacent to the posteromedial aspect of either hemidiaphragm. The warning signs are a typical location and a mass that has a lower density than soft tissue (due to its fat content). ${ }^{(10)}$ In our case, we detected an increased cardiothoracic index, decreased left lung volume, and deviation of the heart and larger mediastinal structures. No mass-like lesion was seen on the patient's chest radiograph. The typical imaging appearance of $\mathrm{BH}$ is one of discontinuity of the posterior or posteromedial diaphragm, with protrusion of the peritoneal or retroperitoneal fat through the defect. Although less common, the colon, small bowel, liver, spleen, or kidneys may also herniate into the thoracic cavity. ${ }^{(5)}$ Herniating organs and the discontinuity of the diaphragm can be better detected with multidetector CT imaging, due to its multiplanar reformatting (MPR) and maximum-intensityprojection (MIP) capabilities. As mentioned, the diagnosis of $\mathrm{BH}$ for our patient was made based on the CTA results. The coronal, axial, and sagittal reformatted CTA images provided us with detailed anatomical knowledge of the diaphragmatic defects and herniated structures in our patient.

$\mathrm{BH}$ may be associated with various congenital anomalies, which are frequently gastrointestinal in nature. These include oesophageal duplication cysts, malrotation of the intestine, Meckel's diverticulum, hepatic hypoplasia, and omphalocele. Pathologies that are cardiac in nature, such as patent ductus arteriosus, patent foramen ovale, ventricular septal defect, preductal aortic coarctation and tetralogy of Fallot, may also be seen. Other disorders that may coexist with $\mathrm{BH}$ are vesicoureteral reflux, undescended testicle, pulmonary hypoplasia, and midline defects like cleft palate and spina bifida. ${ }^{(11,12)}$ With the use of CTA in our case, we identified two loop formations in an elongated descending thoracic aorta and an aberrant right subclavian artery, in addition to the bilateral BHs. Although cardiovascular anomalies associated with $\mathrm{BH}$ have been reported, ${ }^{(11)}$ to the best of our knowledge, this is the first case in the medical literature that describes the occurence of a severely elongated aorta with loop formations and an aberrant subclavian artery, and concomitant $\mathrm{BHs}$. Moreover, there does not appear to be any case report in the literature regarding the concurrent occurence of an aortic loop and an aberrant subclavian artery.

In the differential diagnosis of our patient, we considered diseases that could give rise to the aortic tortuosity seen in our patient, including arterial tortuosity syndrome and Loeys-Dietz syndrome. Both of these syndromes are connective tissue disorders similar to Marfan syndrome in that all of them have a genetic base that is generally detected at an early age. The most striking features of these syndromes are typical craniofacial anomaly that results in a distinctive facial appearance, and elongation, aneurysm and tortuosity of the aorta and supra-aortic arteries. ${ }^{(13,14)}$ As our patient did not have craniofacial anomalies and was of a relatively older age, these syndromes were ruled out as causative factors.

In conclusion, $\mathrm{BH}$ may be associated with a number of congenital anomalies that can be symptomatic and/or highly complex, as shown in our case, in which bilateral $\mathrm{BHs}$ were found to be associated with a severe aortic elongation (with a double loop formation) and an aberrant right subclavian artery. In our opinion, multidetector CT and CTA with MPR and MIP imaging capabilities are reliable imaging modalities that improve the detection of $\mathrm{BHs}$ and any associated anomalies, whether for the purpose of diagnosis or presurgical evaluation.

\section{REFERENCES}

1. Mullins ME, Stein J, Saini SS, Mueller PR. Prevalence of incidental Bochdalek's hernia in a large adult population. AJR Am Roentgenol 2001; 177:363-6.

2. Gale ME. Bochdalek hernia: prevalence and CT characteristics. Radiology 1985; 156:449-52.

3. Salacin S, Alper B, Cekin N, Gulmen MK. Bochdalek hernia in adulthood: a review and an autopsy case report. J Forensic Sci 1994; 39:1112-6.

4. Nitecki S, Bar-Maor JA. Late presentation of Bochdalek hernia: our experience and review of the literature. Isr J Med Sci 1992; 28:711-4.

5. Mei-Zahav M, Solomon M, Trachsel D, Langer JC. Bochdalek diaphragmatic hernia: not only a neonatal disease. Arch Dis Child 2003; 88:532-5.

6. Mallik K, Rodgers BM, McGahren ED. Congenital diaphragmatic hernia: experience in a single institution from 1978 through 1994. Ann Thorac Surg 1995; 60:1331-6.

7. Kırkıl G, Muz MH, Karakoc E. [Bilaterally Located Bochdalek Hernia.] Tur Toraks Dern 2009; 11:147-50. Turkish.

8. Baglaj M, Dorobisz U. Late-presenting congenital diaphragmatic hernia in children: a literature review. Pediatr Radiol 2005; 35:478-88.

9. Sandstrom CK, Stern EJ. Diaphragmatic hernias: a spectrum of radiographic appearances. Curr Probl Diagn Radiol 2011; 40:95-115.

10. Raymond GS, Miller RM, Müller NL, Logan PM. Congenital thoracic lesions that mimic neoplastic disease on chest radiographs of adults. AJR Am J Roentgenol 1997; 168:763-9.

11. Cerilli GJ. Foramen of Bochdalek Hernia. A Review of The Experience at Children's Hospital of Denver, Colorado. Ann Surg 1964; 159:385-9.

12. Guibaud L, Filiatrault D, Garel L, et al. Fetal congenital diaphragmatic hernia: accuracy of sonography in the diagnosis and prediction of the outcome after birth. AJR Am J Roentgenol 1996; 166:1195-202.

13. Loeys BL, Schwarze U, Holm T, et al. Aneurysm syndromes caused by mutations in the TGF-beta receptor. N Engl J Med 2006; 355:788-98.

14. Erdem A, Erol N, Zeybek C, Celebi A. [Arterial tortuosity syndrome in two cases.] Turk Kardiyol Dern Ars 2010; 38:576-9. Turkish. 